\title{
CT Metal Artifact Reduction in the Spine: Can an Iterative Reconstruction Technique Improve Visualization?
}

\author{
(D) A.L. Kotsenas, G.J. Michalak, D.R. DeLone, F.E. Diehn, K. Grant, A.F. Halaweish, A. Krauss, R. Raupach, B. Schmidt, C.H. McCollough,
} and J.G. Fletcher

\begin{abstract}
BACKGROUND AND PURPOSE: Metal-related artifacts from spine instrumentation can obscure relevant anatomy and pathology. We evaluated the ability of CT images reconstructed with and without iterative metal artifact reduction to visualize critical anatomic structures in postoperative spines and assessed the potential for implementation into clinical practice.
\end{abstract}

MATERIALS AND METHODS: We archived CT projection data in patients with instrumented spinal fusion. CT images were reconstructed by using weighted filtered back-projection and iterative metal artifact reduction. Two neuroradiologists evaluated images in the region of spinal hardware and assigned a score for the visualization of critical anatomic structures by using soft-tissue and bone windows (critical structures totally obscured, $n=0$; anatomic recognition with high diagnostic confidence, $n=5$ ). Using bone windows, we measured the length of the most pronounced linear artifacts. For each patient, neuroradiologists made recommendations regarding the optimal use of iterative metal artifact reduction and its impact on diagnostic confidence.

RESULTS: Sixty-eight patients met the inclusion criteria. Visualization of critical soft-tissue anatomic structures was significantly improved by using iterative metal artifact reduction compared with weighted filtered back-projection (median, $1 \pm 1.5$ versus $3 \pm 1.3, P<.001$ ), with improvement in the worst visualized anatomic structure in $88 \%(60 / 68)$ of patients. There was not significant improvement in visualization of critical osseous structures. Linear metal artifacts were reduced from 29 to $11 \mathrm{~mm}(P<.001)$. In $87 \%$ of patients, neuroradiologists recommended reconstructing iterative metal artifact reduction images instead of weighted filtered back-projection images, with definite improvement in diagnostic confidence in $32 \%(22 / 68)$.

CONCLUSIONS: Iterative metal artifact reduction improves visualization of critical soft-tissue structures in patients with spinal hardware. Routine generation of these images in addition to routine weighted filtered back-projection is recommended.

ABBREVIATIONS: IMAR = iterative metal artifact reduction; $\mathrm{wFBP}=$ weighted filtered back-projection; $\mathrm{HU}=$ Hounsfield units

S pinal fusion is commonly performed to treat pain and/or minimize abnormal vertebral motion. In 2011, 488,000 spinal fusion procedures were performed in the United States, a nearly 3-fold increase since 1998. ${ }^{1,2}$ However, despite advances in surgical technique and a proliferation of novel fusion devices and hardware, the rates of the so-called failed back surgery syndrome have not declined, and patients undergoing these procedures often require follow-up imaging. ${ }^{3,4} \mathrm{MR}$ imaging and $\mathrm{CT}$ are both useful in the evaluation of patients after spinal fusion, but both are lim-

Received January 6, 2015; accepted after revision March 11.

From the Department of Radiology (A.L.K., G.J.M., D.R.D., F.E.D., C.H.M., J.G.F.), Mayo Clinic, Rochester, Minnesota; Siemens Medical Solutions (K.G., A.F.H.), Malvern, Pennsylvania; and Siemens Healthcare (A.K., R.R., B.S.), Forchheim, Germany.

Please address correspondence to Amy L. Kotsenas, MD, Department of Radiology, Mayo Clinic, Rochester, MN 55905; e-mail: Kotsenas.amy@mayo.edu; @AmyKotsenas

http://dx.doi.org/10.3174/ajnr.A4416 ited by artifacts related to metallic spinal implants. Misregistration artifacts on MR imaging and beam-hardening artifacts on CT degrade image quality, obscure relevant postoperative anatomy and pathology, and reduce the overall diagnostic confidence in distinguishing normal structures and pathologic findings. ${ }^{5,6}$

On CT, beam-hardening and photon starvation artifacts from metallic fusion hardware frequently compromise visualization of critical anatomic structures and pathologic findings, particularly in the ROI near implanted hardware. Recent publications have presented several postprocessing methods for decreasing the severity of metal implant artifacts in CT..$^{7-11}$ These works describe unique challenges to minimizing metal-related artifacts in different body regions due to differences in local anatomy and in implant composition. ${ }^{12}$ However, only a few of these works focused on the unique challenges of the postoperative spine, ${ }^{12,13}$ and none assessed visualization of the critical anatomic structures that the neuroradiologist must evaluate in the postoperative 
setting. Additionally, prior studies have not systematically assessed how metal artifact reduction images should be incorporated into clinical practice (eg, instead of or in addition to routine images).

In this study, we performed clinical assessment of a prototype iterative metal artifact reduction (IMAR) algorithm (Siemens, Erlangen, Germany) applied to CT data in patients with spinal fusion. The prototype IMAR algorithm makes image-based corrections that aim to minimize artifacts tangential to high-contrast regions on the basis of spatial frequency and recover detail close to the metallic implants, thus theoretically retaining important anatomic information from the original images. Image improvements are refined by using iterative normalization of metal artifact reduction specifically tailored to the postoperative spine. ${ }^{8,14}$ We evaluated the ability of CT images reconstructed with and without IMAR to visualize critical anatomic structures in the postoperative spine and assessed the potential for routine implementation of IMAR into clinical practice.

\section{MATERIALS AND METHODS}

\section{Data Collection and CT Acquisition}

Institutional review board approval was obtained, and informed consent was waived for this retrospective study. Imaging data were collected from July 25, 2012, to August 23, 2013. Inclusion criteria were the following; 1) prior instrumented spine fusion, 2) noncontrast CT imaging of the spine performed at the level of spine fusion, 3) use of a 128-section CT system (Definition Flash; Siemens), and 4) archived CT projection data. All CT imaging was performed by using the routine acquisition parameters of our institution in clinical practice at the time for cervical, thoracic, and lumbar spine CT by using collimation of $128 \times 0.6 \mathrm{~mm}$, pitch of 0.9 , gantry rotation time of 1 second, automatic exposure control tube current setting of 280 or 260 quality reference milliampere-second (for cervical and thoracolumbar protocols, respectively), and a 50-cm scan FOV. Cervical spine scans were performed by using $140 \mathrm{kV}$ (peak), while scans of the lumbar and thoracic spine were performed with $120 \mathrm{kVp}$.

\section{Image Reconstruction}

CT images were reconstructed by a senior research technologist (G.J.M.) from archived projection data by using an off-line computer workstation, with images reconstructed by using a conventional weighted filtered back-projection (wFBP) kernel (B35) and the prototype IMAR algorithm (spine parameters). IMAR was performed by using a vendor-specified "spine" setting, which entails predetermined IMAR reconstruction parameters appropriate for spinal anatomy and hardware. Briefly, for IMAR reconstruction, images are produced by first reconstructing an uncorrected wFBP image, which is segmented into the metal and nonmetal pixels by thresholding. The nonmetal pixels are used to produce a prior image, while the metal pixels are used to generate a metal image. The images are projected back into the sinogram space and are used to normalize and identify the metal projections in the original uncorrected sinogram data. Metal projections data are interpolated, and the sinogram is then denormalized. Highand low-pass-filtered versions of corrected images are then generated. The final images are produced by a weighted combination of the high-pass-filtered images (corrected and uncorrected) with the low-pass-filtered corrected images. This filtering and mixing step is performed iteratively and reduces the blurring of the anatomic structures near the metal object. For both, images of $0.6-\mathrm{mm}$ thickness at $0.6-\mathrm{mm}$ increments were produced with a reconstruction FOV of $50 \mathrm{~cm}$. For the purposes of this retrospective research project, images were displayed to include the entire FOV to assess the whole image for potential artifacts and to delineate their full extent if present.

\section{Image Analysis}

After reconstruction, images were loaded onto an Advantage Windows Workstation (GE Healthcare, Milwaukee, Wisconsin) for viewing. Two Certificate of Added Qualification-certified neuroradiologists (A.L.K., D.R.D.), each with 14 years of experience, performed image evaluation in consensus. Location and type of hardware were identified. Each study was evaluated by viewing axial wFBP and IMAR images side-by-side, first with softtissue window settings (window width, 3700 Hounsfield units [HU]; window level, $600 \mathrm{HU}$ ) and then subsequently with bone window settings (window width, $300 \mathrm{HU}$; window level, $40 \mathrm{HU}$ ). Images were only evaluated in the axial plane without scanogram images or multiplanar reformations.

Visualization of Critical Anatomic Structures. Critical structures were defined as the central canal, the spinal cord, neural foramina, and prevertebral soft tissues. Separate evaluations were performed for evaluation of soft-tissue and osseous structures by using the appropriate window/level display settings. Neuroradiologists evaluated wFBP and IMAR images in a side-by-side fashion for each patient. For both evaluations, a 6-point diagnostic image-quality scale was used $(0=$ critical structures totally obscured, no structures identifiable; 1 = marked artifacts, questionable anatomic recognition of critical structures; 2 = faint anatomic recognition, no confidence in the ability to identify pathology; 3 = anatomic recognition with low confidence in diagnosis; $4=$ anatomic recognition with medium confidence in diagnosis; $5=$ anatomic recognition with high confidence in diagnosis).

For evaluation of soft-tissue structures, the neuroradiologists assigned the overall score on the basis of their impression of softtissue planes and structures. In addition, the neuroradiologists identified the critical anatomic structure that had the worst visualization (ie, greatest anatomic obscuration due to metal or IMAR-related artifacts) and assigned a separate soft-tissue visualization score for this structure by using the same scale. Improvement or worsening in soft-tissue visualization scores was calculated by subtracting the score of the wFBP images from that for the IMAR images so that positive values reflected improvement and negative values reflected degradation.

For evaluation of cortical bone structures, the neuroradiologists examined cortical bone visualization around the region of metal hardware by using the wFBP and IMAR bone window images in a similar fashion and by using the same 6-point scale. Improvement or worsening in visualization of osseous structures between wFBP and IMAR was calculated, as described above.

Objective Artifact Measures. Two objective measures were used to further describe the severity of metal-related artifacts. Verte- 


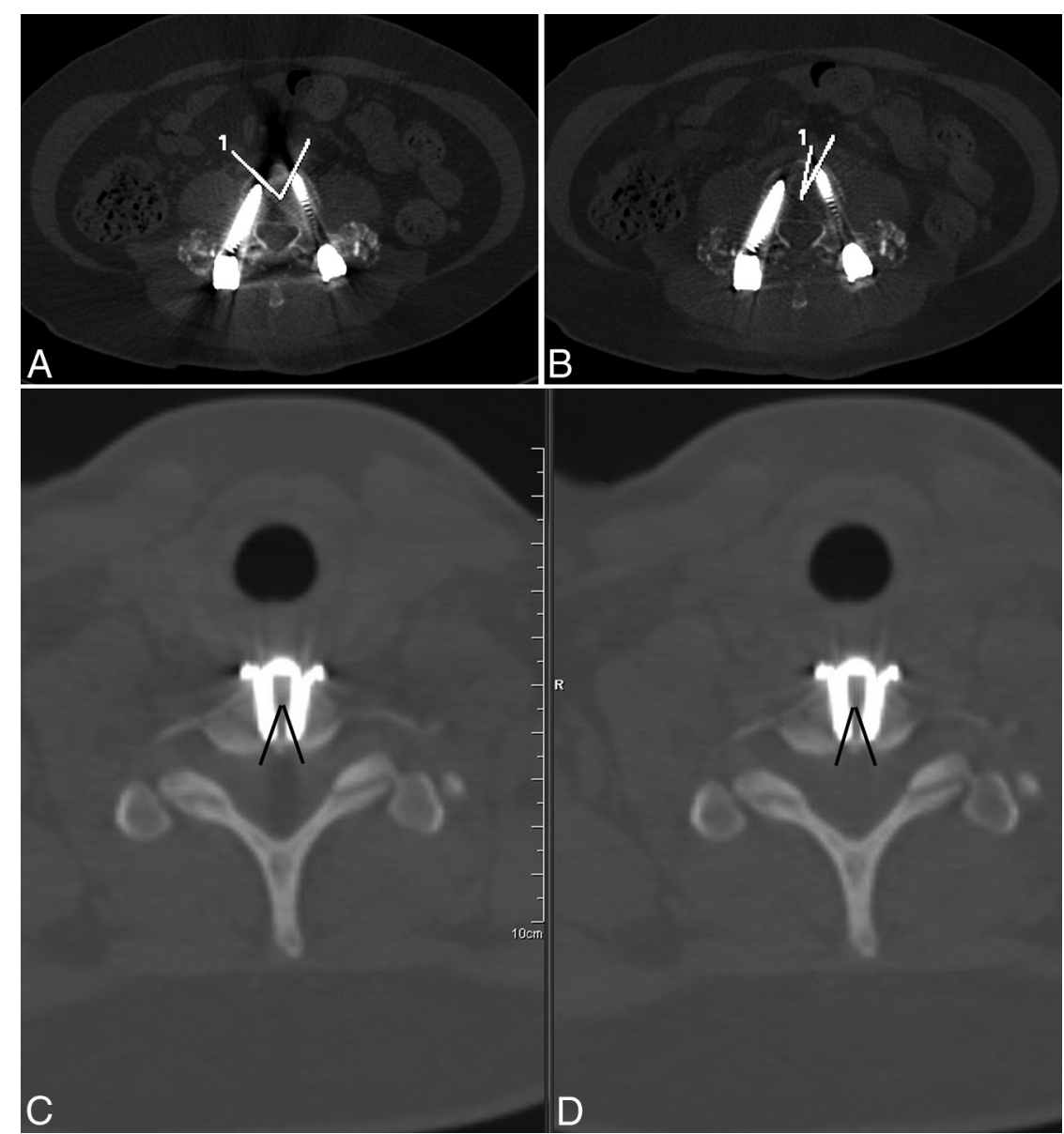

FIG 1. Quantitative measurement describing the maximum extent of vertebral body cortex that was obscured by metal beam-hardening artifacts in 2 patients. The central angle of an angle measurement tool was placed in the middle of the vertebral body, with its rays circumscribing the portions of the vertebral body cortex that were obscured by metal artifacts. The process was repeated for wFBP $(A$ and $C)$ and IMAR $(B$ and $D)$ images. In 1 patient with bilateral screw/rod fixation in the lumbar spine, artifacts from both screws cross at the anterior margin of the vertebral body cortex, resulting in obscuration of a large portion of the anterior cortex $(A)$. There is marked improvement with IMAR in the degree of anterior vertebral body cortical obscuration caused by metal artifacts $(B)$ because only artifacts from the left-sided screw extend to the cortical surface. In another patient with anterior cervical discectomy and fusion ( $C$ and $D)$, there is no change in the degree of vertebral body cortical obscuration as measured by the circumscribed angle.

bral body cortical obscuration was first measured at the level with the greatest beam-hardening artifacts in each patient, by using the same level for each of 2 objective measures. For estimation of vertebral body cortical obscuration, an angle measurement tool was used, with the central angle placed in the middle of the vertebral body as the reference point. The 2 rays forming the angle were drawn so that they circumscribed the portions of vertebral body cortex that were obscured by metal artifacts (Fig 1). This circumscribed angle (or arc, in degrees) was measured for both the wFBP and IMAR images. The improvement in the angular cortical obscuration was calculated by subtracting the angles calculated in the IMAR images from the wFBP images.

A "flame" artifact, reflecting the length of severe beam-hardening parallel to the orientation of spinal hardware (usually a pedicle screw), was also measured. For this measurement, the linear dark band that emanates from the tip of a metal object along its long axis in the imaging plane was measured by first selecting the level of most severe beam-hardening artifacts in each patient.
The extent of the flame artifact was then quantified by measuring the distance (in millimeters) from the tip of the metal object to the end of the linear dark band at the same level on both wFBP and IMAR images (Fig 2). The improvement (decrease) in flame artifact severity was calculated by subtracting the length of the flame artifact on the IMAR images from the length on the wFBP images at the same level so that positive values reflected improvement and negative values reflected degradation.

Recommendation for Clinical Practice. Finally, the neuroradiologist readers assigned recommendations for clinical use of IMAR images and their estimated impact on diagnostic confidence. Impact on diagnostic confidence was a synthesis of the ability to see both critical and other soft-tissue structures, visualization of osseous structures, and introduction of artifacts. These recommendations were made after viewing wFBP and IMAR images in a side-by-side comparison by using both soft-tissue and bone window images for each case. Radiologists were asked to determine their recommendation regarding clinical use of IMAR images with similar future cases as follows: 1 = always generated instead of routine $\mathrm{wFBP}$ images; 2 = always generated in addition to routine $w F B P ; 3=$ only reconstructed when requested; or $4=$ not reconstructed at all. A categoric score was also given for the estimated impact on diagnostic confidence $(0=$ unclear impact, $1=$ probable increase in confidence, 2 = definite increase in confidence, $-1=$ probable decrease in diagnostic confidence, $-2=$ definite decrease in diagnostic confidence).

To ensure that IMAR did not degrade clinically relevant diagnostic information, we retrospectively examined all cases with a description of potential hardware complications at the level of spinal fusion in the clinical radiology reports. In these cases, we compared the visualization of the hardware complication between the wFBP and IMAR images.

\section{Statistical Analysis}

Statistical analysis was performed comparing the soft-tissue and bone visualization scores for critical anatomic structures by using $\mathrm{wFBP}$ and IMAR and for similar comparisons of vertebral body cortical obscuration, by using the 2-tailed Wilcoxon signed rank test. For comparison of flame artifacts between wFBP and IMAR, we used a paired $t$ test because data were normally distributed. A $P$ value $\leq .05$ was considered statistically significant for both sets of comparisons. Descriptive statistics were used to classify spinal hard- 
ware in our cohort, rank improvements in visualization of anatomic structures, and objective measures of artifact severity and to assess recommendations for use of IMAR images in clinical practice.

\section{RESULTS}

Sixty-eight patients with spinal hardware met the inclusion criteria (38 females [56\%]; age range, $17-87$ years). Thirty-nine (57\%) had imaging of the lumbar spine; 21 (31\%), of the cervical spine; and $8(12 \%)$, of the thoracic spine. The radiation dose ranged from 5.9 to $40.7 \mathrm{mGy}$, with a mean dose of $19.6 \mathrm{mGy}$. Thirtyseven patients had posterior rods and pedicle screws, 16 had an anterior fixation plate and screws, 3 had interbody fusion cages, and 12 had other more complex hardware fixation.

\section{Visualization of Critical Anatomic Structures}

The results from the subjective and objective analyses comparing IMAR and wFBP are shown in the Table. The overall soft-tissue visualization scores for critical anatomic structures (median) were $1 \pm 1.5$ for wFBP and $3 \pm 1.3$ for IMAR images $(P<.001)$. The spinal canal was the worst visualized structure in a large majority of patients $(56 / 68 ; 82 \%)$, followed by the prevertebral soft tissues in 7/68 (10\%) and other structures in 5/68 (7\%). The median soft-tissue visualization scores for these worst visualized structures were $0 \pm 1.3$ for wFBP (with a score of zero indicating that the structure is totally obscured), which improved to $3 \pm 1.2$
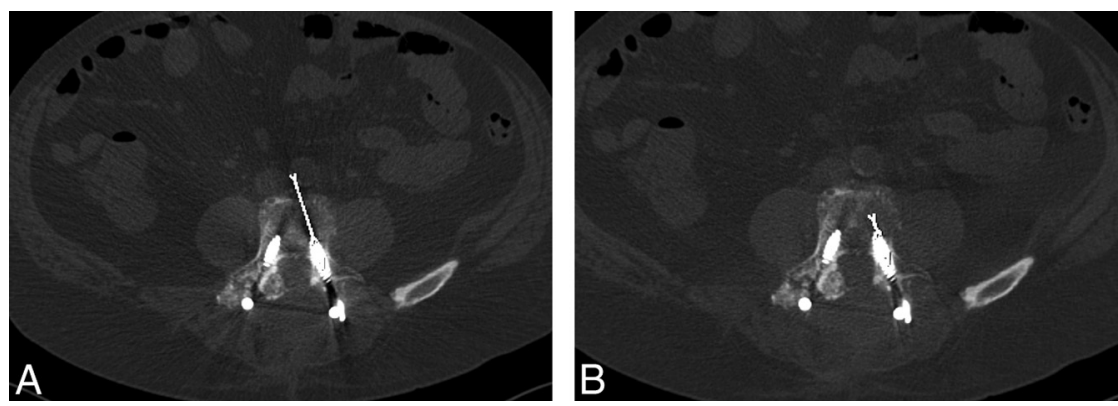

FIG 2. Extent of the flame artifact was quantified by measuring the distance (millimeters) from the tip of the metal object to the end of the linear dark band at the same level on both WFBP (A) and IMAR images $(B)$. Improvement in artifact severity is demonstrated on the IMAR image. for IMAR $(P<.001$; a score of 3 indicating anatomic recognition with a low level of confidence in diagnosis). The mean improvement in overall soft-tissue visualization scores was $1.0 \pm 1.0$, with an improvement of $\geq 1$ point in $55 / 68$ ( $81 \%$ ) patients. The mean improvement in the worst visualized anatomic structure was $1.5 \pm 1.1$, with an improvement of $\geq 1$ point in $60 / 68(88 \%)$ patients (Fig 3). In 11 patients $(16 \% ; 11 / 68)$, the worst visualized anatomic structure improved by at least 3 points. In 4 patients $(6 \%$; $4 / 68$ ), the worst structure improved from a rank of $\leq 2$ with wFBP (no structures identifiable; no confidence in ability to identify pathology) to $\geq 4$ with IMAR (medium-to-high confidence in diagnosis).

The overall bone visualization scores (median) were similar for $w F B P$ and IMAR: $5 \pm 0.5$ and $5 \pm 0.9$, respectively (Fig 4). Only 1 patient $(1.5 \%)$ had worsening of the bone-visualization score. In this patient, complex hardware was present in the thoracic spine causing severe artifacts on both wFBP and IMAR.

In $3 / 68(4.4 \%)$ patients, we observed shadowing at the image periphery somewhere within the reconstructed image stack. None of the images with the shading artifacts contained anatomy evaluated in the study; hence, the artifacts did not affect the study results.

\section{Objective Artifact Measures}

For objective measures of metal-related artifacts, the mean estimate of vertebral body cortical obscuration for wFBP and IMAR images was $7^{\circ}$ and $3^{\circ}$, respectively $(P=.004)$. In 54 of the $68(79 \%)$ patients, the vertebral body cortex was visualized in its entirety on both wFBP and IMAR images. In the 14 patients in whom there was a nonzero value for estimation of vertebral body cortical obscuration, mean angular obscuration was $34^{\circ}$ for wFBP and $15^{\circ}$ for IMAR $(P=.001)$.

Flame-related linear metal artifacts were present in 66/68 (97\%). The mean flame artifact lengths for the wFBP and IMAR images were 29 and $11 \mathrm{~mm}$, respectively $(P<.001)$.

Results from the subjective and objective analyses comparing IMAR and wFBP

\begin{tabular}{|c|c|c|c|c|}
\hline Neuroradiologist Evaluation & wFBP (Median) & IMAR (Median) & Test & $P$ Value \\
\hline \multicolumn{5}{|l|}{ Subjective } \\
\hline Overall soft-tissue visualization score ${ }^{a}$ & $1 \pm 1.50$ & $3 \pm 1.27$ & WSR & $<.001$ \\
\hline Cervical $(n=21)$ & $2 \pm 1.54$ & $4 \pm 1.06$ & WSR & .001 \\
\hline Thoracic $(n=8)$ & & & $N A^{b}$ & $N A^{b}$ \\
\hline Lumbar $(n=39)$ & $1 \pm 1.24$ & $3 \pm 1.15$ & WSR & $<.001$ \\
\hline Soft-tissue structure with worst artifacts, visualization score ${ }^{a}$ & $0 \pm 1.34$ & $3 \pm 1.19$ & WSR & $<.001$ \\
\hline Cervical $(n=21)$ & $1 \pm 1.53$ & $3 \pm 1.20$ & WSR & .001 \\
\hline Thoracic $(n=8)$ & & & $N A^{b}$ & $N A^{b}$ \\
\hline Lumbar $(n=39)$ & $0 \pm 1.18$ & $2 \pm 1.04$ & WSR & $<.001$ \\
\hline Bone (cortex) visualization score & $5 \pm 0.49$ & $5 \pm 0.87$ & WSR & .02 \\
\hline Cervical $(n=21)$ & $5 \pm 0.36$ & $5 \pm 0.30$ & WSR & .16 \\
\hline Thoracic $(n=8)$ & & & $N A^{b}$ & $N A^{b}$ \\
\hline Lumbar $(n=39)$ & $5 \pm 0.54$ & $5 \pm 0.16$ & WSR & $<.001$ \\
\hline \multicolumn{5}{|l|}{ Objective } \\
\hline Vertebral body cortical obscuration (in degrees) & $7 \pm 17$ & $3 \pm 12$ & PTT & $<.001$ \\
\hline Vertebral body cortical obscuration, when present $(n=15)$ (degrees) & $34 \pm 22$ & $13 \pm 24$ & PTT & $<.001$ \\
\hline Length of flame artifacts $(\mathrm{mm})$ & $29 \pm 18$ & $11 \pm 7$ & PTT & $<.001$ \\
\hline
\end{tabular}

Note:-NA indicates not applicable; PTT, 2-tailed paired $t$ test; WSR, Wilcoxon signed rank test.

a Structures evaluated in the region of metal fixation hardware included the central canal, spinal cord, neural foramina, and prevertebral soft tissues.

${ }^{\mathrm{b}}$ A subgroup analysis of thoracic spine cases was not performed due to the few cases in this group. 

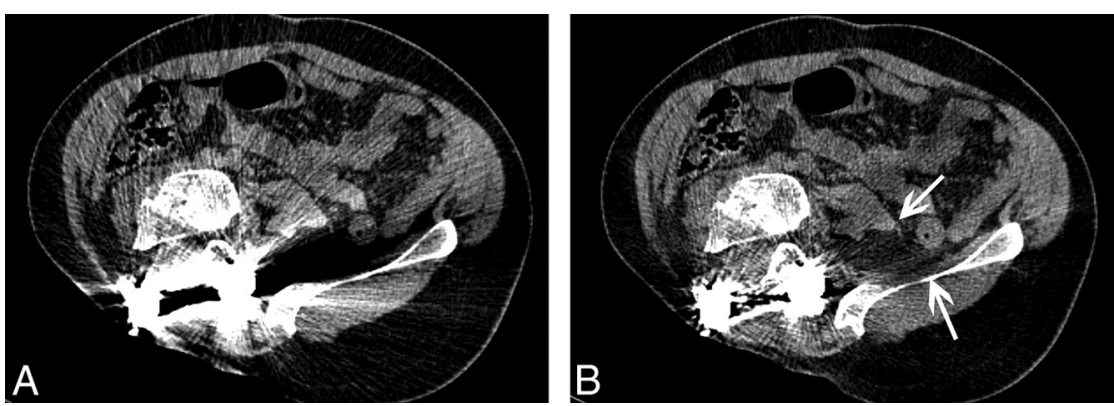

FIG 3. $W F B P(A)$ and IMAR $(B)$ images in a 33-year-old man status post right hemipelvectomy for resection of a fibrosarcoma. IMAR improves visualization of the psoas and iliacus muscles (arrows), the retroperitoneal fat, and the spinal canal to exclude tumor recurrence.
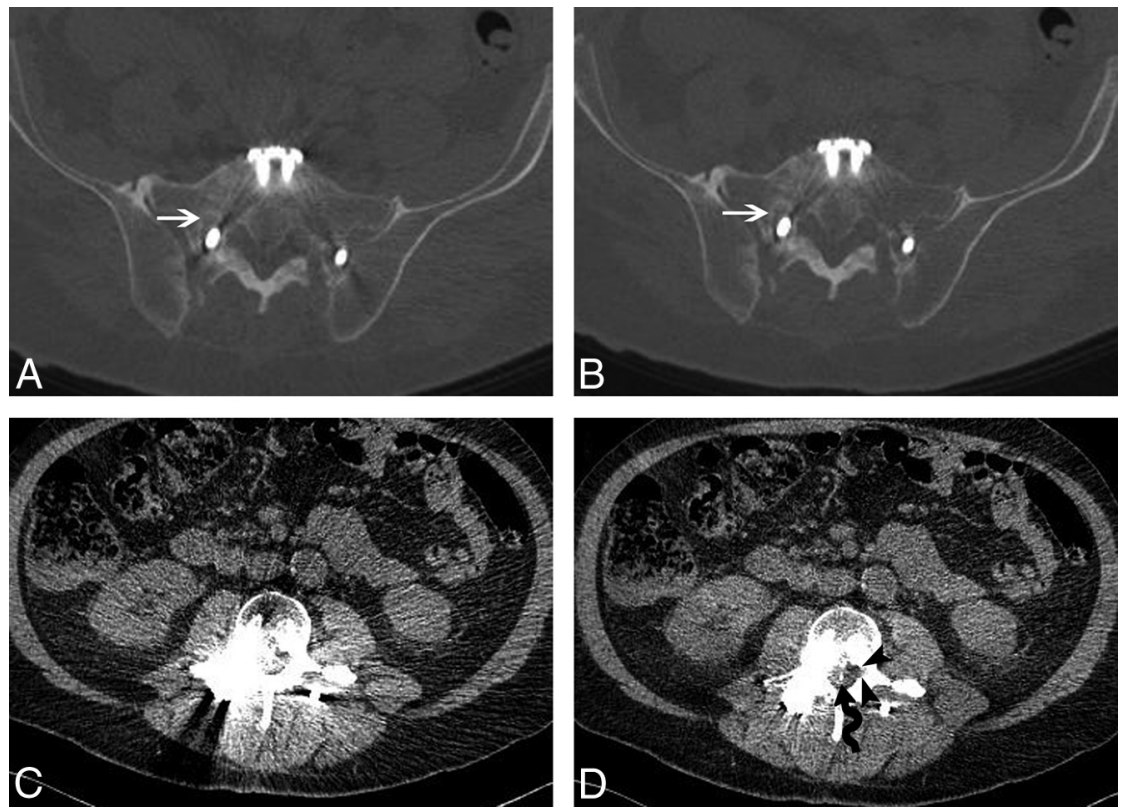

FIG 4. A 65-year-old man status post L3-to-S1 pedicle screw and rod fixation. WFBP $(A)$ and IMAR $(B)$ images at the S1 level using bone window settings demonstrate lucency about the right S1 screw, consistent with hardware loosening (white arrows). At the L3 level, the central canal and lateral recesses are obscured by artifacts on wFBP $(C)$ image with soft-tissue window settings. $\operatorname{IMAR}(D)$ image with soft-tissue window settings at the same level demonstrates a retained wire from a prior spinal cord stimulator (wavy arrow) and improved visualization of the left lateral recess (black arrowheads).

\section{Recommendation for Clinical Practice}

In most patients (87\%; 59/68), the neuroradiologists wanted IMAR images instead of wFBP images; in an additional 10\% (7/ 68 ), IMAR images were requested in addition to wFBP images. These cases included those in which soft-tissue artifacts remote from the spine were identified. In 3\% (2/68) of patients, IMAR images were deemed necessary only if specifically requested. Both of these patients had complex, multilevel fusions. When asked about the impact of IMAR images on diagnostic confidence, the readers estimated a definite increase in confidence in clinical diagnosis in 22/68 (32\%) patients, a probable increase in $25 / 68$ (37\%) patients, unclear impact on diagnostic confidence in $21 / 68$ $(31 \%)$ patients, and probable or definite decrease in none $(0 \%)$.

Seven percent $(5 / 68)$ of patients had a hardware complication at the level of the spinal fusion based on retrospective review of clinical radiology reports (3 with hardware loosening, 1 with a pedicle screw extending beyond the medial pedicle margin, and 1 with migration of an interbody fusion cage). In these 5 patients, IMAR images were thought to display the hardware complications as well as the FBP images (Fig 4).

\section{DISCUSSION}

The presence of metallic structures such as screws, rods, and dental fillings in CT images may cause severe artifacts due to beam-hardening, photon starvation, and the presence of sharp gradients in the sinogram data. Metal objects can generate streaks or dark areas in the image and obscure low-contrast neural structures of interest in the spinal canal or in adjacent paravertebral soft tissues. Our pilot study demonstrated significant improvement in visualization of critical anatomic structures such as the spinal canal and adjacent paravertebral soft tissues by using a prototype IMAR reconstruction technique. Additionally, IMAR reduced the extent of linear "flame" artifacts. In those cases in which the vertebral body cortex was obscured, IMAR improved cortical visualization. Neuroradiologist readers recommended reconstruction of IMAR images routinely in clinical practice in $>90 \%$ of cases (and in $>80 \%$, they recommended reconstruction of IMAR without wFBP images). Neuroradiologists thought that the use of IMAR images definitely improved diagnostic confidence in $32 \%$ of patients. These results are important because they demonstrate that this technology may be a useful method for reducing metal artifacts on CT images in the ever-growing population of patients with spinal fixation hardware, improving the diagnostic benefit of wFBP images, particularly in the evaluation of critical soft-tissue anatomic structures.

Most studies evaluating metal artifact reduction algorithms have evaluated orthopedic hardware in phantoms or in a heterogeneous mix of patients (often with none having spinal-fixation hardware). Similar to our study, however, Wang et $\mathrm{al}^{13}$ evaluated 18 patients with spinal fusion and metal hardware, using dualenergy, virtual monochromatic kiloelectron volt images and metal artifact reduction algorithms. They used subjective 5-point image-quality and diagnostic interpretability scales in addition to measuring the width of pedicle screws as an objective measure of metal artifacts. These investigators found that their approach resulted in improved image quality and diagnostic interpretability but that metal artifact reduction degraded pedicle screw shape. In contrast, we imaged a larger number of patients with different types of spinal fixation hardware and performed a visual analysis 
of critical anatomic structures that may be affected by postoperative complications. Furthermore, we performed a side-by-side assessment of wFBP and IMAR images across our cohort to determine how such algorithms should be integrated into routine clinical practice. We submit that while IMAR images are sufficient to replace wFBP images in $>80 \%$ of patients, both wFBP and IMAR images should be routinely reconstructed for the minority of patients in whom unusual artifacts can be caused by postprocessing with the IMAR algorithm itself (Fig 4). Routine reconstruction of IMAR images is warranted, given the definite or probable increase in diagnostic confidence in more than half of patients, usually on the basis of improved visualization of softtissue structures.

Several recent proposals for metal artifact reduction have used dual-energy CT with virtual monochromatic kiloelectron volt images in addition to metal artifact reduction algorithms. ${ }^{7,14}$ However, the effectiveness of artifact reduction by using monoenergetic imaging appears to be dependent on implant material and size. ${ }^{7,9,12,13}$ Our work used a method for metal artifact reduction that can be employed using conventional single-energy CT acquisition with thresholds and other settings potentially altered to adjust for different types and location of metal implants. Others have previously described a 1D linear interpolation to improve CT sinogram data, ${ }^{15,16}$ but such approaches have not been put into clinical practice until recently, primarily due to the high computational time requirements. ${ }^{17}$ Recently, the linear interpolation approach was improved by adding a normalization process (ie, "normalized metal artifact" reduction) that can be performed quickly and efficiently. ${ }^{10}$ The IMAR prototype used in this study is yet a further improvement to these existing algorithms. We herein test this new approach for the first time in a large set of patients with spinal hardware by using an off-line computer workstation.

Some limitations of our study should be acknowledged. First, as described above, in this study, the projection data gathered during each patient's examination were exported to an off-line station where the reconstruction with the prototype IMAR algorithm was performed. This delay in workflow would not likely be clinically acceptable on a routine basis. We did not record the off-line image reconstruction time for each case because CT scanner image reconstruction systems are much more powerful than the off-line workstation. We anticipate that IMAR implementation in a scanner image reconstruction system will reconstruct several IMAR images per second. Second, evaluation of wFBP and IMAR images was performed in a side-by-side fashion, so the readers could readily ascertain which image set was created by using the IMAR algorithm. This methodology was required to allow detection of subtle differences in visualization of soft-tissue structures between the 2 techniques and quantification of the most severe artifacts at the same anatomic level and to understand the nature and frequency of artifacts caused by the IMAR algorithm itself. Our CT system can reconstruct images by using sinogram-affirmed iterative reconstruction, another vendor-specific iterative reconstruction technique, but we did not generate a third set of images by using sinogram-affirmed iterative reconstruction for this analysis because sinogram-affirmed iterative reconstruction itself may cause artifacts and the goal in this study was not to reduce noise but to reduce metal artifacts. Additionally, we only evaluated noncontrast images.

The implications of using IMAR for examinations in which intravenous or intrathecal contrast (ie, myelography) is administered will require further investigation. Finally, we used visualization of critical anatomic structures and estimation of diagnostic confidence as a surrogate for detection of postoperative pathology; an increase in diagnostic confidence may not translate into clinical relevance. We only assessed axial images and did not view scanogram images or multiplanar reformats as would be routine for our clinical practice. While our results suggest that abnormalities such as nerve root or spinal cord compromise or the presence/absence of soft-tissue tumor recurrence may be determined with higher confidence by using IMAR, we did not design our study to address this hypothesis. Given that IMAR reconstruction may take time to perform, the true clinical utility of IMAR remains uncertain despite our findings. Prospective assessment of the ability of IMAR to identify clinical findings will be a focus of future work.

\section{CONCLUSIONS}

The IMAR prototype improves anatomic visualization of critical soft-tissue structures in the postoperative spine and reduces metal artifacts by both subjective and objective measurement, resulting in improved diagnostic confidence in the large majority of patients with spinal fusion hardware.

Disclosures: Amy L. Kotsenas—RELATED: Grant: Siemens, ${ }^{*}$ Comments: Siemens created the prototype iterative metal artifact reduction technique under study. David R. DeLone-RELATED: Grant: Siemens, ${ }^{\star}$ Comments: Mayo Clinic receives a grant from Siemens, creator of the metal artifact reduction prototype under study; Travel/Accommodations/Meeting Expenses Unrelated to Activities Listed: Siemens, Comments: Mayo Clinic-Siemens Collaboration Summit 2014. Katharine Grant-RELATED: Other: Siemens, Comments: I am a full-time employee of Siemens USA (includes stock/stock options). Ahmed F. Halaweish—UNRELATED: Employment: Siemens. Andreas Krauss-OTHER RELATIONSHIP: employee of Siemens. Rainer Raupach — UNRELATED: Employment: Siemens; Patents (planned, pending, or issued): Siemens; Stock/Stock Options: Siemens. Bernhard Schmidt—UNRELATED: Employment: Siemens. Cynthia H. McCollough—RELATED: Grant: Siemens, ${ }^{*}$ Comments: This work was part of a larger sponsored research grant to evaluate new CT technology. I am the Principal Investigator of this grant. During the course of the study, I had no direct access to any study data or results; Support for Travel to Meetings for the Study or Other Purposes: Siemens, ${ }^{\star}$ Comments: Travel support was provided for me to report to Siemens the results of the overall technology research grant that I have with them; Provision of Writing Assistance, Medicines, Equipment, or Administrative Support: Siemens, Comments: I am the Principal Investigator of a technology research grant with Siemens. A portion of the funds are for administrative support; UNRELATED: Patents (planned, pending or issued): Siemens, ${ }^{*}$ Comments: Mayo Clinic and I have intellectual property rights for technology outside the work performed here for which Siemens has purchased licensing rights; Royalties: Siemens, ${ }^{*}$ Comments: Mayo Clinic and I have intellectual property rights for technology outside the work performed here for which Siemens has purchased licensing rights. Joel G. Fletcher-UNRELATED: Grants/Grants Pending: Siemens,* Comments: I am co-investigator on a grant from Siemens, which provided the prototype metal artifact reduction software for this study. *Money paid to the institution.

\section{REFERENCES}

1. Rajaee SS, Bae HW, Kanim LE, et al. Spinal fusion in the United States: analysis of trends from 1998 to 2008. Spine (Phila $\mathrm{Pa} 1976$ ) 2012;37:67-76 CrossRef Medline

2. Weiss AJ, Elixhauser A, Andrews RM. Characteristics of Operating Room Procedures in U.S. Hospitals, 2011: Healthcare Cost and Utilization Project Statistical Brief \#170. Rockville: Agency for Healthcare Research and Quality; 2014 
3. Chan CW, Peng P. Failed back surgery syndrome. Pain Med 2011; 12:577-606 CrossRef Medline

4. Young PM, Berquist TH, Bancroft LW, et al. Complications of spinal instrumentation. Radiographics 2007;27:775-89 CrossRef Medline

5. Stradiotti P, Curti A, Castellazzi G, et al. Metal-related artifacts in instrumented spine: techniques for reducing artifacts in CT and MRI—state of the art. Eur Spine J2009;18(suppl 1):102-08 CrossRef Medline

6. White LM, Buckwalter KA. Technical considerations: CT and MR imaging in the postoperative orthopedic patient. Semin Musculoskelet Radiol 2002;6:5-17 CrossRef Medline

7. Lee YH, Park KK, Song HT, et al. Metal artefact reduction in gemstone spectral imaging dual-energy $\mathrm{CT}$ with and without metal artefact reduction software. Eur Radiol 2012;22:1331-40 CrossRef Medline

8. Lell MM, Meyer E, Schmid M, et al. Frequency split metal artefact reduction in pelvic computed tomography. Eur Radiol 2013;23: 2137-45 CrossRef Medline

9. Meinel FG, Bischoff B, Zhang Q, et al. Metal artifact reduction by dual-energy computed tomography using energetic extrapolation: a systematically optimized protocol. Invest Radiol 2012;47:406-14 CrossRef Medline

10. Meyer E, Raupach R, Lell M, et al. Normalized metal artifact reduction (NMAR) in computed tomography. Med Phys 2010;37:5482-93 CrossRef Medline
11. Meyer E, Raupach R, Lell M, et al. Frequency split metal artifact reduction (FSMAR) in computed tomography. Med Phys 2012;39: 1904-16 CrossRef Medline

12. Guggenberger R, Winklhofer S, Osterhoff G, et al. Metallic artefact reduction with monoenergetic dual-energy $\mathrm{CT}$ : systematic ex vivo evaluation of posterior spinal fusion implants from various vendors and different spine levels. Eur Radiol 2012;22:2357-64 CrossRef Medline

13. Wang Y, Qian B, Li B, et al. Metal artifacts reduction using monochromatic images from spectral CT: evaluation of pedicle screws in patients with scoliosis. Eur J Radiol 2013;82:e360-366 CrossRef Medline

14. Morsbach F, Wurnig M, Kunz DM, et al. Metal artefact reduction from dental hardware in carotid CT angiography using iterative reconstructions. Eur Radiol 2013;23:2687-94 CrossRef Medline

15. Kalender WA, Hebel R, Ebersberger J. Reduction of CT artifacts caused by metallic implants. Radiology 1987;164:576-77 CrossRef Medline

16. Mahnken AH, Raupach R, Wildberger JE, et al. A new algorithm for metal artifact reduction in computed tomography: in vitro and in vivo evaluation after total hip replacement. Invest Radiol 2003;38: 769-75 CrossRef Medline

17. Beister M, Kolditz D, Kalender WA. Iterative reconstruction methods in X-ray CT. Phys Med 2012;28:94-108 CrossRef Medline 